Proceedings

Juntendo Medical Journal

2016. 62 (Suppl 1), 44-49

\title{
Sportology to Prevent Locomotive Syndrome
}

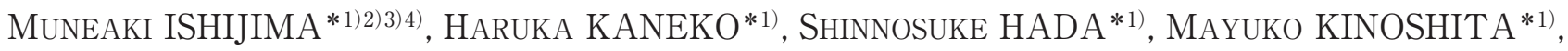 \\ MASAYOSHI ISHIBASHI*3), RYO SADATSUKI*1), LIZU LIU*12), HITOSHI ARITA*1), \\ JUn SHIOZAWA*1), ANWARJAN YUSUP*1), Yoshitomo SAITA*1), Yuji TAKAZAWA*1), \\ SHUICHI MACHIDA*4)5), HisASHI NAITO*4)5), HiROSHI IKEDA*1), \\ ERI ARIKAWA-HIRASAWA*4)6), YASUNORI OKADA*3), KAZUO KANEKO*12)3)
}

*1) Department of Medicine for Orthopaedics and Motor Organ, Juntendo University Graduate School of Medicine, Tokyo, Japan, *2) Sportology Center, Juntendo University Graduate School of Medicine, Tokyo, Japan, *3) Department of Pathophysiology for Locomotive and Neoplastic Diseases, Juntendo University Graduate School of Medicine, Tokyo, Japan, *4) Center of Innovation (COI) program, Juntendo University Graduate School of Medicine, Tokyo, Japan, *5) Graduate School of Health and Sports Science, Juntendo University, Chiba, Japan, *6) Research Institute for Diseases of Old Age, Juntendo University Graduate School of Medicine, Tokyo, Japan

\begin{abstract}
"Locomotive syndrome" is defined as a condition associated with being restricted in one's ability to walk or lead a normal life owing to a dysfunction in one or more of the parts of the locomotion system, including the muscles, bones, joints, cartilage or the intervertebral discs. This syndrome especially refers to those elderly who have come to need nursing care services because of problems with the locomotive organs, or who have conditions which may require them to have such services in the near future. The recent epidemiological studies revealed that the one fourth of the reasons for requiring special assistance or nursing care in elderlies is currently the locomotive disorders. Osteoarthritis of the knee (knee OA) or hip and the spinal canal stenosis due to spondylosis are the three major locomotive disorders those are related to the requiring special assistance or nursing care in elderlies. We are trying to elucidate the mechanistic insight into the pathophysiology of knee OA and osteoporosis by receiving several supports, such as the COI program by a MEXT and the Juntendo Sportology Center under the concept of "Sportology", as we believe to be able to contribute directly the concept of "locomotive syndrome" by these actions.
\end{abstract}

Key words: locomotive syndrome, knee osteoarthritis, osteoporosis, spinal canal stenosis, fragility fracture

\section{Locomotive syndrome}

Japan has been developed to be a leading country for not only the average life span but also the healthy life expectancy worldwide. The healthy life expectancy means the number of years we can expect to live in good health without any special assistance or nursing care. Global healthy life expectancy in Japan (73.4 yo) is no. 1 in worldwide in both female $\left(75.6\right.$ yo) and male $(71.1 \text { yo })^{1)}$.
Therefore, Japan faces a future as the most elderly society humankind has ever known.

The Japanese Orthopaedic Association proposed the concept of "locomotive syndrome" in 2007. It is defined as a condition associated with being restricted in one's ability to walk or lead a normal life owing to a dysfunction in one or more of the parts of the locomotion system, including the muscles, bones, joints, cartilage or the intervertebral discs. This syndrome especially refers to those

Corresponding author: Muneaki Ishijima

Department of Medicine for Orthopaedics and Motor Organ, Juntendo University Graduate School of Medicine

2-1-1 Hongo, Bunkyo-ku, Tokyo 113-8421, Japan

TEL: +81-3-3813-3111 Fax: +81-3-3813-3428 E-mail: ishijima@juntendo.ac.jp

The $2^{\text {nd }}$ Congress, International Academy of Sportology〔Held on Sep. 12, 2015〕

〔Received Dec. 18, 2015] 


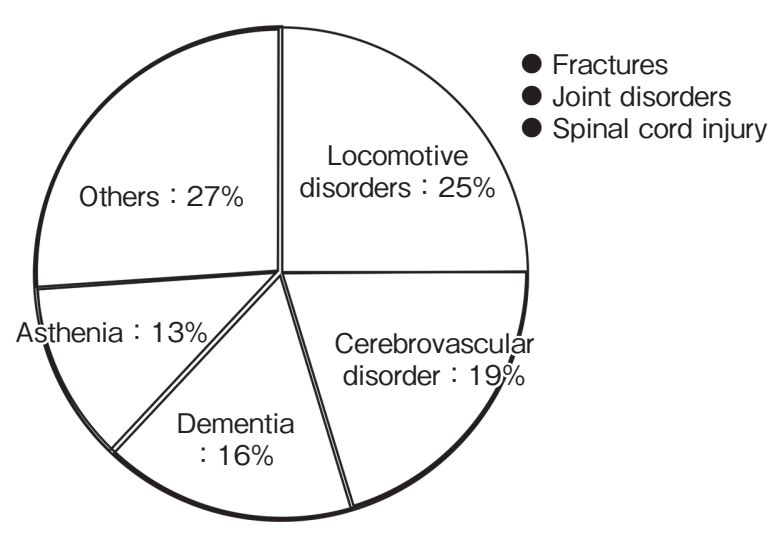

Figure-1 Causes for nursing care in elderlies in Japan (Annual Health, Labour and Welfare Report 2013-2014. Ministry of Health, Labour and Welfare; $2015^{2)}$ )

elderly who have come to need nursing care services because of problems with the locomotive organs, or who have conditions which may require them to have such services in the near future.

The recent epidemiological studies revealed that the one fourth of the reasons for requiring special assistance or nursing care in elderlies is currently the locomotive disorders (Figure-1) ${ }^{2)}$. The three major locomotive disorders those are related to the requiring special assistance or nursing care in elderlies are the osteoporotic fragility fractures, osteoarthritis of the knee or hip and the spinal canal stenosis due to spondylosis (Figure-2). The prevalence of these disorders is increased dependent upon aging. In addition, it has also been revealed that elderlies are suffering from not only one of these three locomotive disorders but also two or all of these disorders, simultaneously, and the complication rate of these three locomotive disorders in elderlies is increasing depending upon aging $(\text { Figure }-3)^{3)}$. These locomotive organ disorders worsen as signs go unheeded. Steps need to be taken today to prevent locomotive syndrome and to extend the healthy life expectancy of people living today, so that individuals can continue to be mobile for life (Figure-4).

Researches for the pathophysiology of the osteoarthritis of the knee (knee OA) under the concept of "Sportology"

Osteoarthritis of the knee (knee OA) is one of the representative age-related chronic motor organ diseases responsible for the locomotive syndrome.
$\mathrm{OA}$ is an age-related progressive joint disease, which is characterized primarily by cartilage degradation ${ }^{4)}$. OA is an increasingly important public health concern, as the prevalence of the disease is increasing with the aging of society. The ideal management of knee $\mathrm{OA}$ is illustrated as a sequential, pyramidal approach (Figure-5) ${ }^{5}$. While it has been estimated that there are 25 million people with radiographic knee $\mathrm{OA}$, it has been speculated that eight million have knee pain ${ }^{6}$. Among the patients with painful knee OA, eighty-five thousand cases of total knee arthroplasty (TKA) are currently being performed each year in Japan. For example, the concept of "locomotive syndrome" should therefore be promoted so that the patients with knee OA who either need or don't need TKA can be identified more clearly from the perspective of the locomotive syndrome, and to allow for the earlier identification of patients with symptomatic knee OA to prevent the development of locomotive syndrome by providing adequate pain relief.

For future development of the disease-modifying drugs in knee OA, further studies are required to elucidate precisely the process of the disease progression. Under the concept of "Sportology" to prevent the locomotive syndrome and the support from the Juntendo Sportology Center, we have been tried to elucidate the pathophysiology of knee OA. For instance, we revealed, for the first time, that the degenerative changes, detected by $\mathrm{T} 2$ mapping on magnetic resonance imaging (MRI), and the morphological changes, detected on MRI, of the femoral articular cartilage showed a greater degree of deterioration than those of both the tibial- and patellar- articular cartilage in the patients with early stage knee OA (Figure-6) ${ }^{7)}$ This is considered to be unique and important information for obtaining a better understanding of the disease processes, which has never previously been elucidated using classical radiographs.

We have also investigated the metabolic changes of the articular cartilage in men in early forties without radiographic OA changes and symptom, including pain. The participants of this study were the subjects in the Juntendo Sportology Core study, which was been conducted since 2011. The radiographic medial knee joint space width (JSW) of the subjects showed the normal distribution. The 


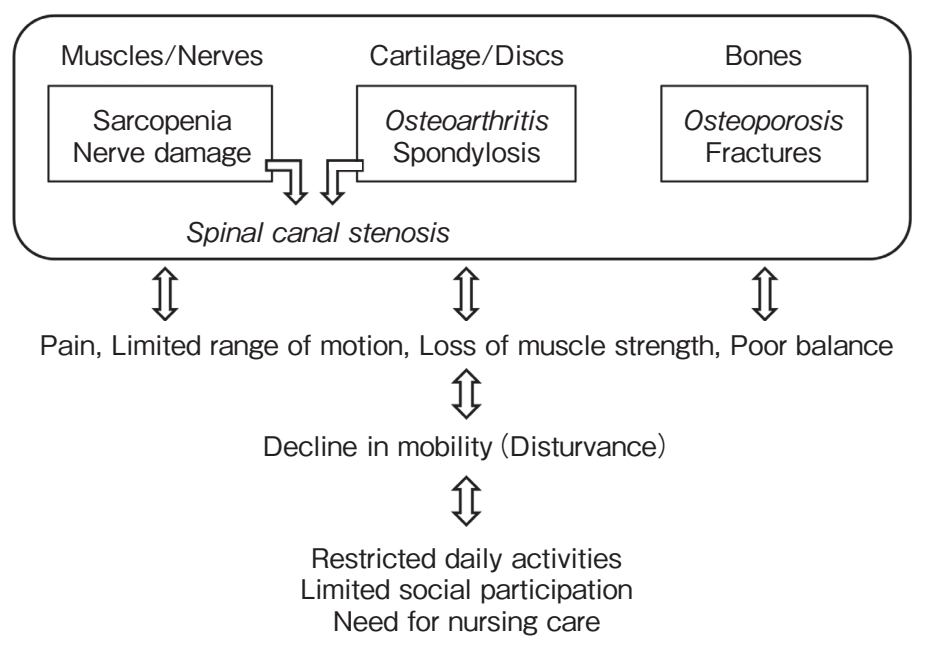

Figure-2 Locomotive syndrome: A conceptual diagram (Nakamura K: J Orthop Sci, 2011; 16: 489-491 ${ }^{8)}$ )

\begin{tabular}{lccc}
\hline & Total & Male & Female \\
\hline Either one & 44.7 & 21.0 & 26.0 \\
\hline Two among three & 24.7 & 9.9 & 14.8 \\
\hline All three & 5.4 & 1.1 & 4.3 \\
\hline & & & (millions)
\end{tabular}
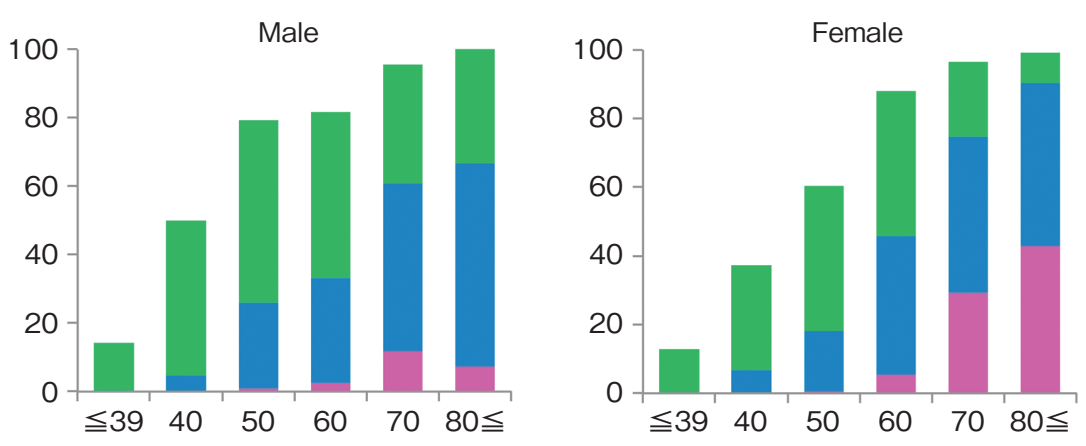

Figure-3 Prevalence of knee osteoarthritis, lumbar spondylosis and osteoporosis in Japanese men and women (Yoshimura N, et al: J Bone Miner Metab, 2009; 27: 620-628 ${ }^{3)}$ )

serum levels of procollagen II C-terminal propeptide ( $\mathrm{SP}$ II $\mathrm{CP}$ ), which is a cartilage synthesis biomarker, and cartilage type II collagen cleavage by collagenase $(\mathrm{sC} 2 \mathrm{C})$ and the urinary levels of cartilage type II collagen C-telopeptide (uCTX- II), cartilage destruction biomarkers, were measured. Among these three cartilage biomarkers, a multiple linear regression analysis with adjustments for age and BMI showed significant positive correlations between the radiographs JSW and the sP II CP level ( $\beta$ : $0.404, p=0.007$ ), suggesting the reduction of cartilage synthesis was associated with the lower JSW, that is one of the typical signs of OA. Thus, the lower cartilage synthesis, rather than cartilage destruction, was associated with the radiographic joint space narrowing of the knee joint in men in early forties without any symptom related to knee $\mathrm{OA}$. 


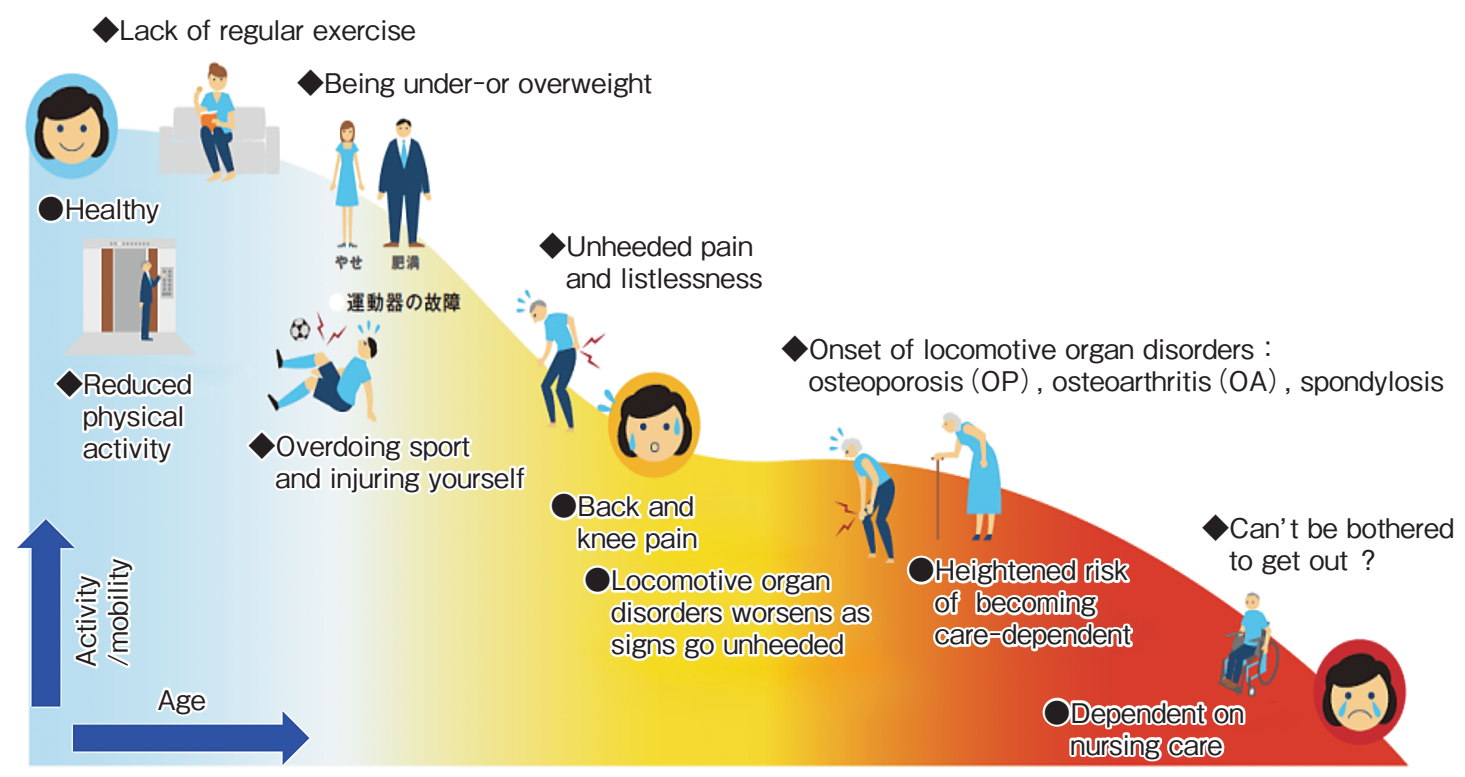

Figure-4 Relationship between the development of locomotive syndrome and the mobility of people (Locomotive Syndrome. 2013: Accessed at https://locomo-joa.jp/en/index.pdf.)

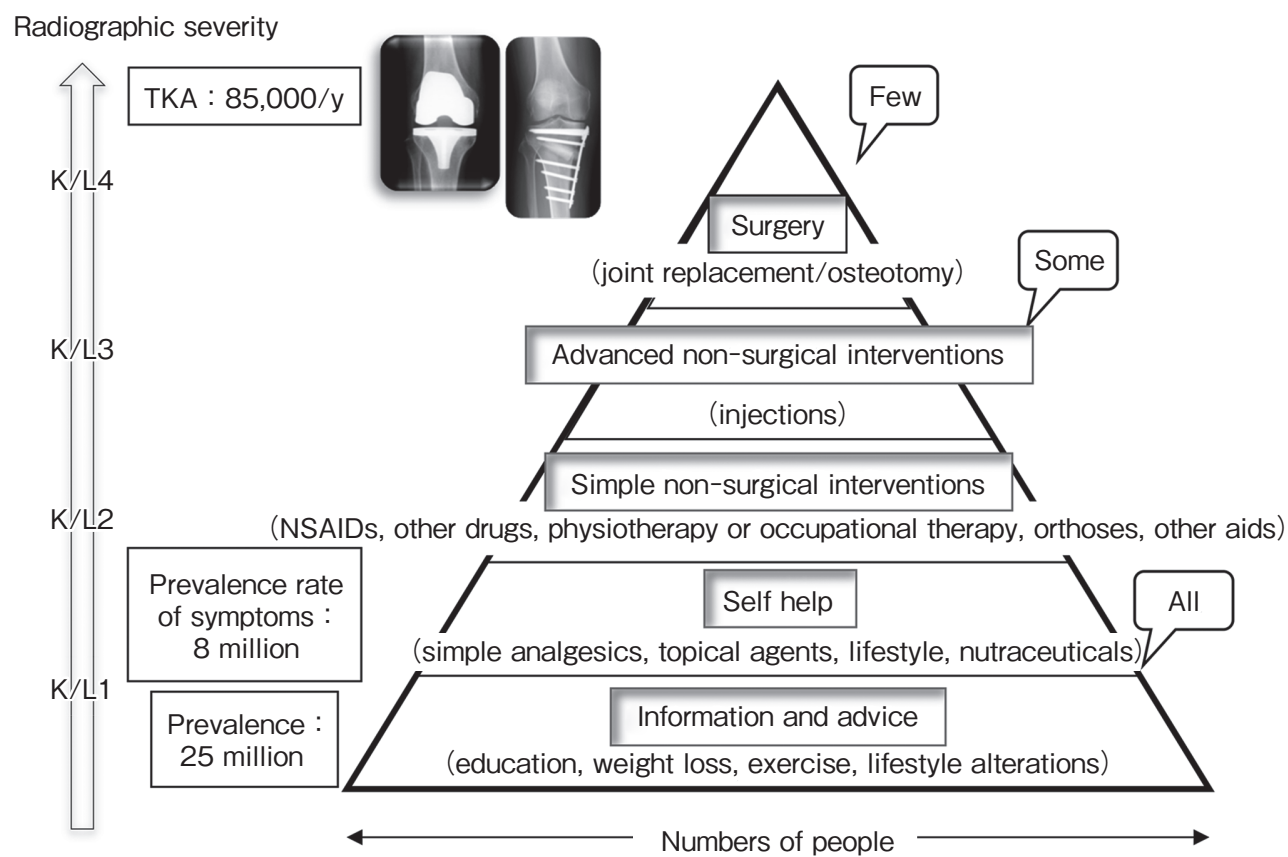

$\mathrm{K} / \mathrm{L}:$ Kellegren-Lawrence grade

Figure-5 Principles of the management of knee OA

Suggested sequential, pyramidal approach to disease management.

(Dieppe PA, et al: Lancet, 2005; 365: 965-9735) 


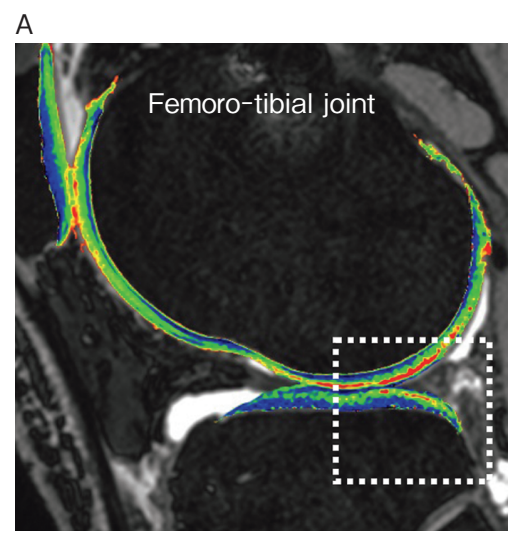

B
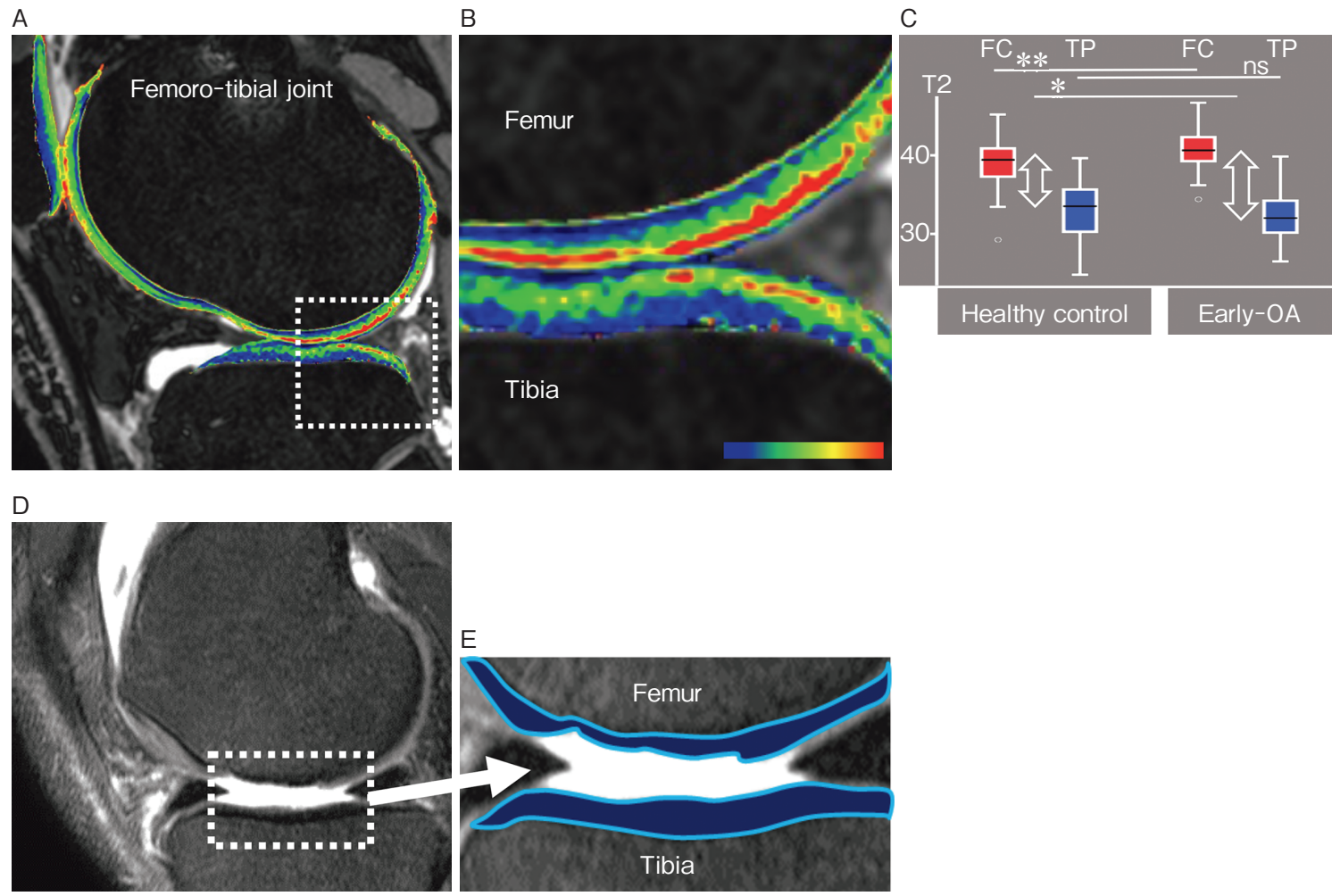

Figure-6 The early stage OA-induced degenerative and morphological changes between the femoral- and the confronting tibial- or patellar- sides within the knee join were not the same

A. T2 mapping image of the sagittal section of the knee joint. B. Enlargement of the articular cartilage of (A). C. Comparison of theT2 levels of articular cartilage in early-stage knee OA : femoral side $>$ tibial side $(\mathrm{p}<0.001)$, odd ratio: $3.6(95 \% \mathrm{CI}: 1.8-6.9, \mathrm{p}<0.0001)$. 3T MRI image of the sagittal section of the knee joint. D. Comparison of the cartilage lesions in early-stage knee OA : femoral side $>$ tibial side $(\mathrm{p}<0.001)$, odd ratio: 1.9 (95\%CI: 1.0-3.5, p=0.026). E. Enlargement of the articular cartilage of (D).

(Hada S, et al: Osteoarthritis Cartilage, 2014; 22: 1583-15897)

\section{Researches for the locomotive syndrome supported by the Center of Innovation (COI) program}

We are also supported by the Center of Innovation (COI) program from the Japan Science Technology Agency (JST) and the Ministry of Education, Culture, Sports, Science and Technology (MEXT), Japan, which is a large-scale and university-industry collaborated R\&D platform in which universities concentrate all their powers on innovative research issues and corporations lead the commercialization of their achievements. In the diagnosis and treatment of knee OA, information for gait of patients with knee OA is important. Although the gait of patients with knee OA can be measured using motion capture technology, the devices currently available require a huge space to monitor and scan the patient's motion, making it difficult to use in the clinical setting. Under the support from the COI program and the collaboration with the researchers of both HITACHI Ltd and

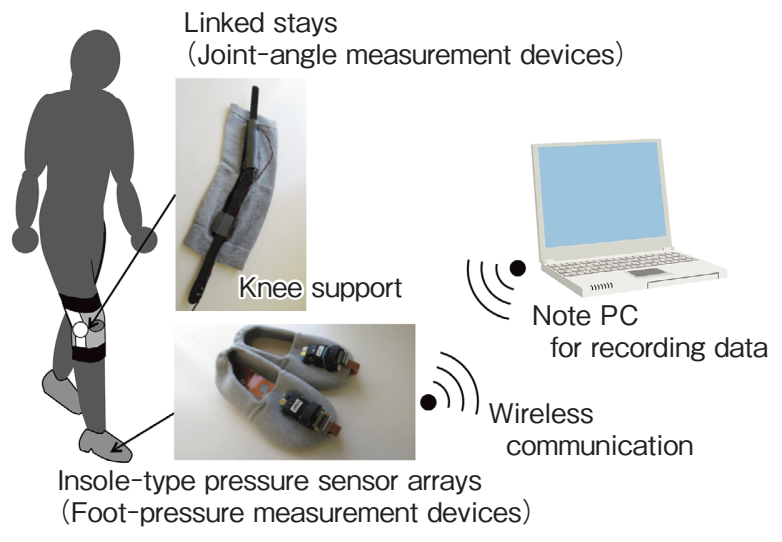

Figure-7 Novel motion capture technology developed by HITACHI Ltd. to monitor the walking ability of patients with knee OA in the clinical setting

(Kaneko H, et al: Juntendo Medical Journal, 2014; 60: 525)

Juntendo University Graduate School of Health and Sports Science, we have established a novel motion capture technology to monitor the walking ability of patients with knee OA in the clinical setting. This device consists of linked stays and insole-type 
pressure sensor arrays. The stays have inertial sensors (accelerometers and gyroscopes) to measure the hip joint angles, and potentiometers to measure the knee joint angles. The pressure sensor arrays which are inserted into the shoes measure the balance (center of pressure) of the patients while they walk. These light weight (350 g including shoes $)$ and small $(3 \times 5 \times 2 \mathrm{~cm})$ devices can measure the gait and send the results to a $\mathrm{PC}$ without the need for a video monitoring system (Figure-7). We demonstrated the possibility that the novel miniaturized motion capture technology could monitor the walking ability of patients with knee $\mathrm{OA}$ in the clinical setting. We also demonstrated that the range of motion of hip joint and the walking stride were the factors that related early postoperative walking recovery by analyzing gait analysis before and after TKA in end-stage knee OA.

\section{Sportology to prevent locomotive syndrome}

There is an urgent need to improve the understanding of the pathophysiology of the diseases, such as knee OA and osteoporosis, which are responsible for "locomotive syndrome". Further studies are required to facilitate the understanding of the "locomotive syndrome" widely and the concept "Sportology" could be helpful to succeed this proposition.

\section{Acknowledgement}

We deeply appreciate the support by a Center of
Innovation (COI) program from the Japan Science Technology Agency (JST) and the Ministry of Education, Culture, Sports, Science and Technology (MEXT) and by a High Technology Research Center Grant from the MEXT.

\section{References}

1) Collaborators GDaH: Global, regional, and national disability-adjusted life years (DALYs) for 306 diseases and injuries and healthy life expectancy (HALE) for 188 countries, 1990-2013: quantifying the epidemiological transition. Lancet, 2015; 386: 2145-2191.

2) Annual Health, Labour and Welfare Report 2013-2014. Tokyo: Ministry of Health, Labour and Welfare; 2015.

3) Yoshimura N, Muraki S, Oka H, et al: Prevalence of knee osteoarthritis, lumbar spondylosis, and osteoporosis in Japanese men and women: the research on osteoarthritis/osteoporosis against disability study. J Bone Miner Metab, 2009; 27: 620-628.

4) Felson DT: Clinical practice. Osteoarthritis of the knee. N Engl J Med, 2006; 354: 841-848.

5) Dieppe PA, Lohmander LS: Pathogenesis and management of pain in osteoarthritis. Lancet, 2005; 365: 965973.

6) Muraki S, Oka H, Akune T, et al: Prevalence of radiographic knee osteoarthritis and its association with knee pain in the elderly of Japanese population-based cohorts: the ROAD study. Osteoarthritis Cartilage, 2009; 17: 1137-1143.

7) Hada S, Kaneko H, Sadatsuki R, et al: The degeneration and destruction of femoral articular cartilage shows a greater degree of deterioration than that of the tibial and patellar articular cartilage in early stage knee osteoarthritis: a cross-sectional study. Osteoarthritis Cartilage, 2014; 22: 1583-1589.

8) Nakamura K: The concept and treatment of locomotive syndrome: its acceptance and spread in Japan. J Orthop Sci, 2011; 16: 489-491. 